\title{
A Mobile Portal for Pharmaceutical Services
}

\author{
http://dx.doi.org/10.3991/ijim.v7i1.2352 \\ Mohammed Otair ${ }^{1}$, Aiman Al-Refaei ${ }^{1}$, Bashar Sarayreh ${ }^{1}$ \\ ${ }^{1}$ Amman Arab University, Amman, Jordan
}

\begin{abstract}
This paper initiated to develop a WAP based ordering system. The paper extends the existing WEB enabled sales order capability to include a WAP service. Sales Representative Place the customer order during the customer visit to reduce time to order from second day to instant. Reduce paper work and costs associated with the recording of sales orders. In the Pharmacist Convenience to order, to check his /her account balance anytime anywhere, Get info on promotional items, and place orders.
\end{abstract}

Index Terms - component; Mobile Portal; WAP; Pharmaceutical services; Smart-phones.

\section{INTRODUCTION}

Mobile applications that are used in business field become very efficient nowadays. The mobile has many essential functions such as: multimedia messaging service (MMS), short messaging service (SMS) and voice service. In addition to, it has been developed to allow people make email service, video calls and WAP browsing. Smartphones which they have operating systems compared to laptops and personal computers have been brought to market recently. This new generation of mobile devices creates the mean of ease to use advanced service and business applications especially advance email features and specified business applications with enhanced manner of security; such as mobile banking, mobile booking services, and much more applications that can be used by mobile WEP or WAP. The development of mobile networks and mobile internet bandwidth causes a huge expansion in using mobile applications in business field.

Mobile devices can be used for trading through internet; it is called Mobile commerce (m-commerce) which lets people buy or sell goods, services, or even information using one of using one method of the wireless technology methods. The expansion of using smartphones, mobile devices and the development of mobile network nowadays increase the wide spread of $\mathrm{m}$-commerce applications [8]. Using smartphones and mobile devices can make the $\mathrm{m}$ commerce very easy to be applied and used without extra cost. Therefore, users can pay their digital payments easily and securely. While, for long time ago, the mobile device security was difficult to overcome; however, producing more secure mobile applications can help a lot to overcome the obstacle.

The aim of this paper is to create a model for mobile business sector in the context of the pharmaceutical application as a strategic tool which helps in creating a sustainable competitive advantage through the implementation of an effective mobile business portal.

\section{MOBILE BUSINESS AND MOBILE COMMERCE}

Today, mobile are very important role in human life. It is considered as an efficient way which opens new markets for those who are trying to get benefits of such technology that allows them expand their goals and activities. Mobile technology has influenced personal communications as it is reliable, suitable and low-cost. The Internet users are 2.2 billion users in 2011 as in [10, 11]. Wireless communication and mobile internet technology can help users to have access of information which is related to work and personal life anytime, anywhere including all mobile services which provide professional process to manage people's activities and mobile operations and they also provide M-commerce. On the other hand, specific factors like limited bandwidth, multiple functionalities and small screen display of handheld devices can delay the access [5]. Wireless communication become very popular since, more Wireless Application Protocol (WAP) have been improved by using Wireless Markup Language (WML) which is used to translate Hypertext Markup Language (HTML) pages for those who use WAP to most data that is already designed for users of personal computer via screens of their mobile phones [3].

The main of focus of the most common implementations of mobile business sector lies in exchanges goods, services or information between a business corporate and its consumers (B2C), between two or multiple businesses (B2B), and between a business corporate and its employees (B2E), and other advanced implementations including: consumer-to-government (C2G), consumer-to-consumer (C2C), or between professionals and a business (B2P.) Sometimes it could be the exchange between machines which is called (M2M). The previous dimensions are based on incorporating parameters of the business paradigm including integration form, market strategy, initiator, and government. The initiator is entity acting in the network or value chain which picks the initiative to make an integration progress which can be downstream from a sales representative or it can be upstream to a customer. It is a plausible assumption that from either a sales representative or a customer position proceed is taken from a dominant position in the value chain. As for the any new entrant to enter the value chain, the market should be fragmented on the two sides [6]. It is significant in M-Business marketing to develop awareness of the emerging market opportunities to improve innovation, and to adopt and provide the management of long-term relationships between customers, such as gathering data about market components from different sources, and sharing it via the 
functional units/departments within an organization, this phenomena is called 'Marketing intelligence'. However, in M-business and M-commerce, the main focus was recently directed to dynamic between sales representative, managements and customer [7].

To make M-Business a success, it has to meet the multidimensional customer needs such as: providing new versions of characterized as easy and convenient to use and facilitating the executions transactions within a short period of time. An example of that is the airline sector; there would be executable away from airports, a mobile check-in process [6].

\section{PHARMACEUTICAL MOBILE PORTAL}

The first generation initiatives implemented recently by many Pharmaceutical sectors emphasize the potential of M-Business to distinguish their products and services to decrease process costs. Pharmaceutical industries are adopting, M-Business to provide sales representative with information and as a tracking system. The Characteristic approaches concentrate on efforts to develop a delocalization culture amongst communities who have not traditionally had to transfer information [4]. M-Business is considered as a significant source of competitive advantage and certain abilities for making and transferring information as a main element of organizational advantage. It is based on business processes of organizations and the employee's skills that give a company a lot of capabilities which can provide customers with a service, product or information [1].

Pharmaceutical companies implement Operational Mobile Business (OMB) applications that provide significant enabling capabilities to improve front-office operations and analysis. Providing customized and efficient sales, marketing, and services via multichannel cooperation means that the firm will be experiencing high service costs, long sales cycles, or high customer turnover which help the organizations to resolve the troubleshoots while it is driving decreasing costs and revenue growth. An example of that is the state-of-the-art technology providing by potential M-business enabling service engineers and sales persons to have a full access to full history of all interaction for the customer [2].

\section{A FRAMEWORK FOR UNDERSTANDING MOBILE WORK}

Mobile networking technologies like digital data The WAP specifications that extend and leverage, such as URLs, HTTP, scripting, XML, and digital networking can be increased and spreaded by using the WAP specifications. Figure 1 shows a conceptual WAP framework for under-standing the potential development of mobile work in organizations, which we will refer to as the Mobile Work Model (MWM). The diagram shows three levels [9].

- Mobile level: Mobile browser is a wireless application.

- Operator level: WAP gateway, which acts as an HTTP proxy.

- Organization level: the Application and Database Server.
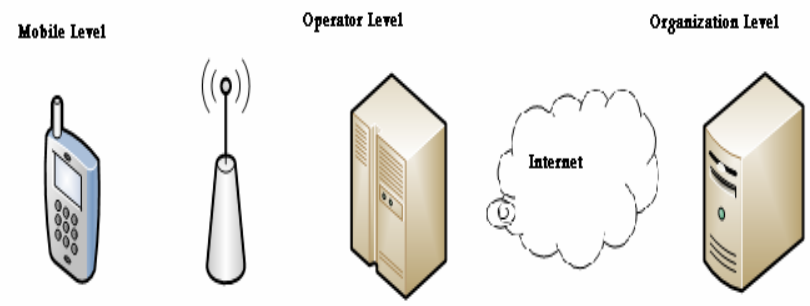

Figure 1. Framework for mobile work Model (MWM)

Fig (1) shows the framework of mobile work Model (MWM) which provides a general framework for deploying and developing ambulant services and applications which can be accessed by WAP-compliant mobile machines extended to include point-to-point services between mobile device and business applications.

In wireless environment, the Wireless Application Protocol (WAP) is used as a standard for the data oriented applications. In spite of the development of some components of the WAP package, still it lacks the whole general structure which integrates software parts of both wireless contexts and the Internet in a direct link or way.

\section{IMPLEMENTATION}

This Section discusses some implementation techniques for the proposed architecture and outline the WAP System including, the activity diagram that explains each Form, functionality and layout the technical aspects of the WAP system which is serving as a communication media between the user and Data Base. On this system, we utilized the Microsoft technologies (Microsoft Visual C\#.Net, and MS SQL Server)

This section composed Graphical User Interface (GUI), and Procedure explanation which is composed of two sections:

- GUI explanation for its interaction with the rest of the system

- Functions and procedure explanation

Those above-mentioned points will form the implementation WAP Application.

Fig (2) introduces the use-cases of the system combined with the activity diagrams explaining the flow for each case to provide a clear functional view of the WAP System.

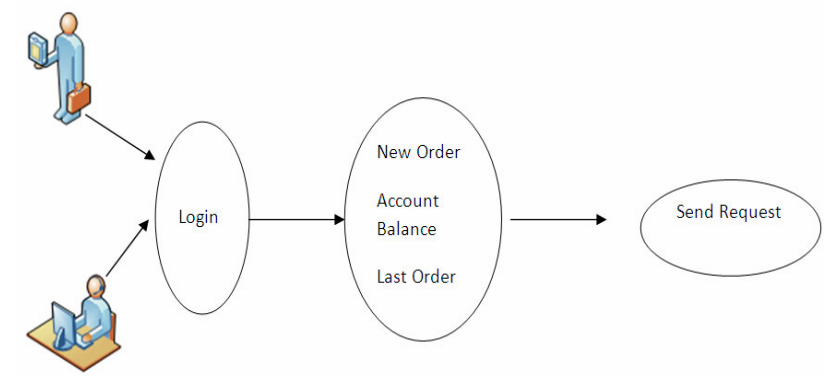

Figure 2. Use Cases of the developed System 


\section{SERVER SIDE}
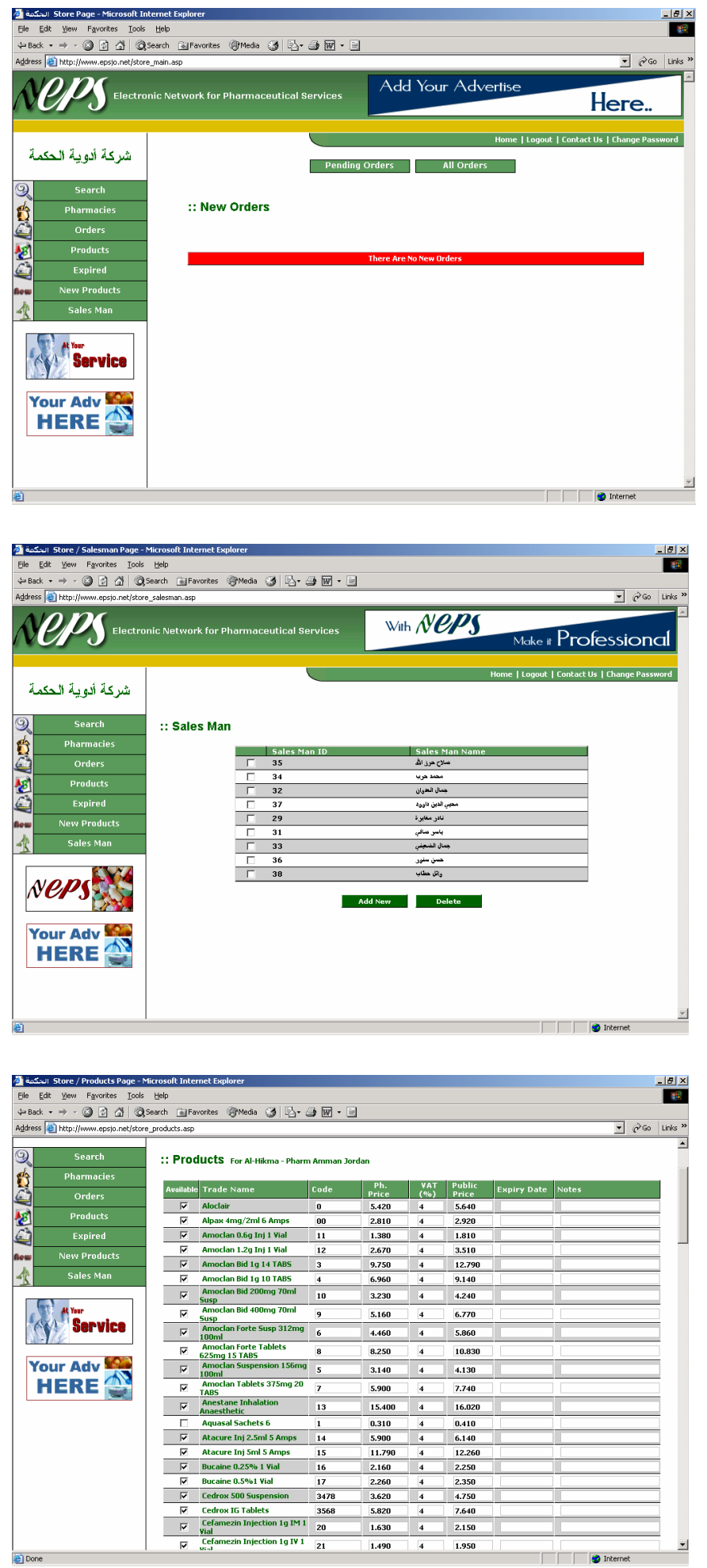

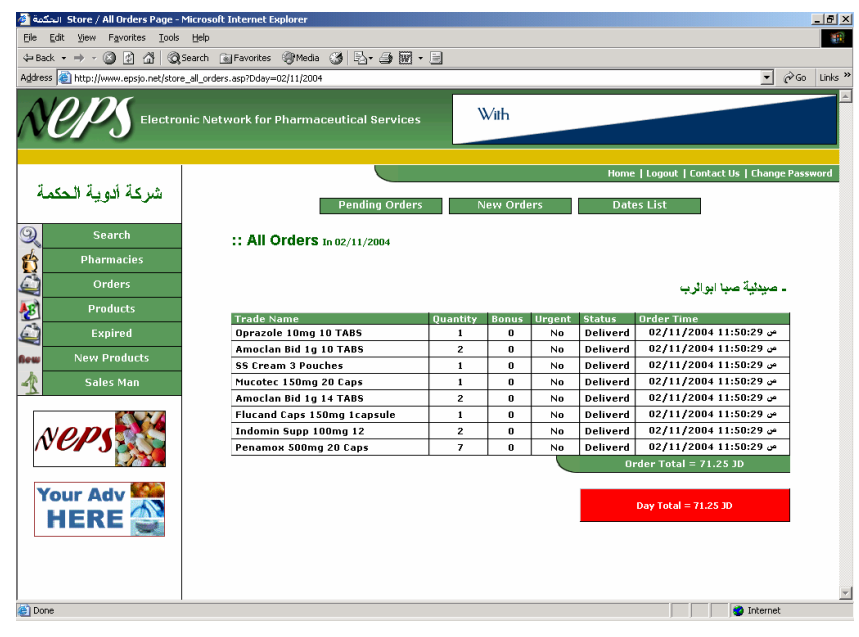

\section{CLIENT SIDE}

\section{A. Main Page (A)}

This describes the selection of language needed for user before login to the system.

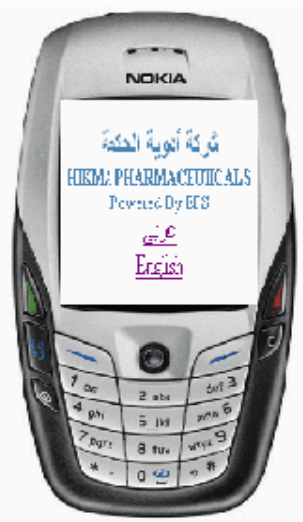

\section{B. $\operatorname{Login}(B)$}

This describes the process of accessing to the WAP system by providing credentials (i.e. User Name and Password) which will verified the system and user (Sales representative, Pharmacy) base granted access on her/his credentials.

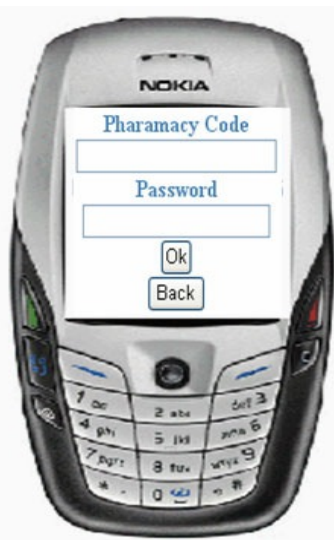




\section{Search Pharmacy (C)}

This describes the operation of searching a specific pharmacy; Sales representatives can use the pharmacy code to see the main screen, or search for the pharmacy then view the main screen.

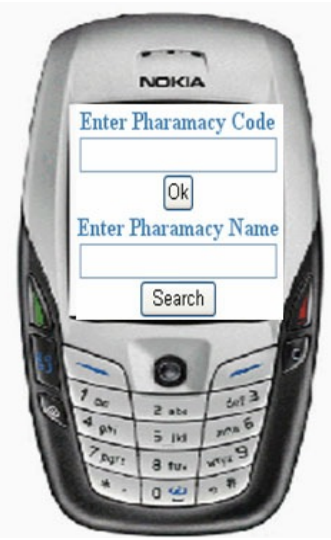

\section{Search Item (D)}

This describes the operation of searching a specific item; this search based on more than one criterion as described below:

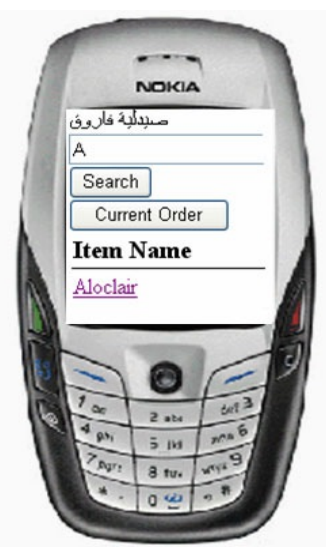

\section{E. List Items (E)}

This provides information on how to view different lists of items (Drugs).

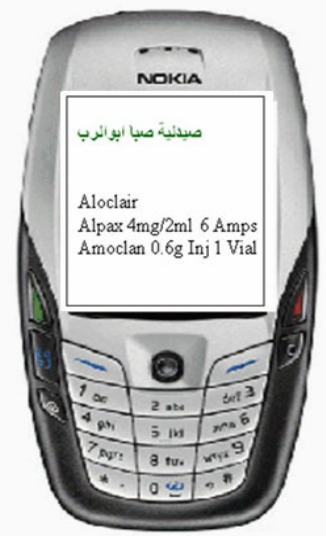

\section{F. $\quad$ Place Orders $(F)$}

This used to place orders; this can do several times based on the user's (Sales representative, Pharmacy) needs.

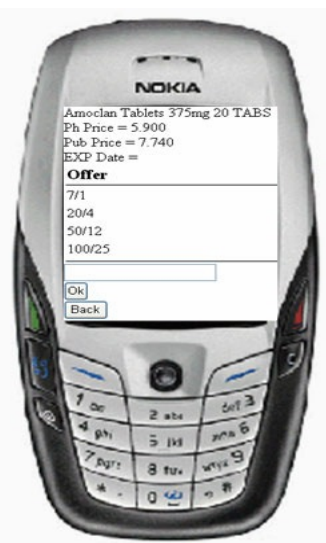

\section{G. Send $\operatorname{Order}(G)$}

This makes the user (Sales representative, Pharmacy) able to send his/her orders.

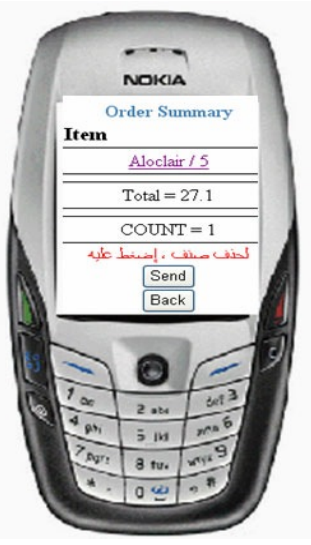

\section{H. Account Balance (H)}

This makes the user (Sales representative, Pharmacy) able to request an account balance for a specific pharmacy.

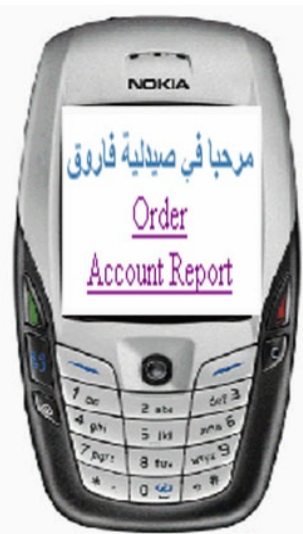

\section{Last Order (I)}

This describes how the user (Sales representative, Pharmacy) can see his/her orders during the last 7 days. 


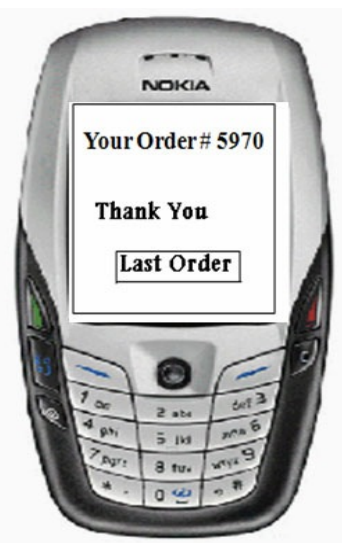

\section{CONCLUSION}

Mobile implementations are used in many fields. They become the essential stream that can be applied throughout many sectors such as Pharmaceutical. Mobile applications play an important role in increased by using the responsiveness and efficiency of pharmaceutical companies.

\section{REFERENCES}

[1] Adam, O. Institute for Information Systems, German Research Center for Artificial Intelligence, Germany ,"Managing interorganizational business processes using an architecture for $\mathrm{M}$ business scenarios". International Conference on Mobile Business 2005.

[2] Glissmann,S.;Kolbe,L.M.; Brenner, W. ".Switzerland Performance measurement of mobile marketing in multi-channel", Environments. International Conference on Mobile Business 2005.

[3] Gwo-Jen H., Judy C.R. T., Yu-San H ,I-WAP: An Intelligent WAP Site Management. , IEEE Transactions on Mobile Computing, April-June, Vol. 1, No. 2, pp. 82-95, 2002.

[4] Hammami Ramzi, Yannick Frein, Atidel B. Hadj-Alouane," THE RELEVANT FEATURES OF SUPPLY CHAIN DESIGN IN THE DELOCALIZATION CONTEXT" 19th International Conference on Production Research, 2007.

[5] P. Candace Deans, E-Commerce and M-Commerce Technologies book, 2005 .

[6] Pagiavlas, Notis; Stratmann, Marzel; Marburger, Peter; Young, Seth "BUSINESS COMPREHENSIVE MARKETING STRATEGIES OR MERELY IT EXPENSES? A CASE STUDY OF THE US AIRLINE INDUSTRY", Journal of Electronic Commerce Research, VOL. 6, NO.3, p 251-261, 2005.

[7] Rollins, Minna \& Halinen, Aino," Customer Knowledge Management Competence: Towards a Theoretical Framework", Proceedings of the 38th Hawaii International Conference on System Sciences -2005 .

[8] Sai Ho Kwok and Robert Chi, "DIGITAL RIGHTS MANAGEMENT FOR MOBILE COMMERCE USING WEB SERVICES” - Journal of Electronic Commerce Research, VOL 7, NO.1, 2006.

[9] Wireless Internet Architecture course material, Manonmaniam Sundaranar University,india , http://niv.ac.in/WirelessInternet Architecture.pdf

[10] http://www.internetworldstats.com/stats.htm

[11] http://www.jeff-peters.com/2011/04/infographic-2011-mobileusage-statistics

\section{AUTHORS}

Dr. Mohammed Otair is with the Dept. of Computer Information Systems, Amman Arab University, Amman, Jordan, otair@aau.edu.jo.

Dr. Aiman Al-Refaei is with the Dept. of Computer Information Systems, Amman Arab University, Amman, Jordan, aiman.alrefaei@aau.edu.jo.

Dr. Bashar Sarayreh is with the Dept. of Management Information Systems, Amman Arab University, Amman, Jordan, bsarayreh@gmail.com.

Received 13 November 2012. Published as resubmitted by the authors 20 December 2012. 